\title{
The Chosen Few: Parallel Library Reaction Methodologies for Drug Discovery Supporting Information
}

Amanda W. Dombrowski,* Ana L. Aguirre, Anurupa Shrestha, Kathy A. Sarris, and Ying Wang*

Advanced Chemistry Technologies Group, AbbVie, Inc., North Chicago, Illinois 60064, United States

Supporting Information Table of Contents

Table S1. Amide Coupling Conditions Used in Library Synthesis.............................................................

Table S2. Suzuki Coupling Conditions Used in Library Synthesis ...............................................................

Table S3. Common C-N Coupling Library Reaction Conditions Used with Different Substrates........................7 
Table S1. Amide Coupling Conditions Used in Library Synthesis

\begin{tabular}{|c|c|c|c|c|}
\hline Reagent 1 & Reagent 2 & Reagent 3 & Base & Solvent(s) \\
\hline & CDI & & DBU & DCE \\
\hline & CDI & & DBU & DME \\
\hline & CDI & & & THF \\
\hline $\mathrm{PS}^{\mathrm{PPh}} \mathrm{P}_{3}$ & $\mathrm{Cl}_{3} \mathrm{CCN}$ & DMAP & TEA & THF \\
\hline PS-DCC & HOBt & Si-carbonate & TEA & DMA \\
\hline \multirow{45}{*}{ PS-DCC } & HOAt & & TEA & DMA \\
\hline & HATU & & TEA & DMA \\
\hline & HATU & & TEA & DMF \\
\hline & HATU & & TEA & DMA/THF \\
\hline & HATU & & TEA & DMA/dioxane \\
\hline & HATU & & TEA & DMA/DMSO \\
\hline & HATU & & TEA & DCM \\
\hline & HATU & & TEA & DMA/ACN \\
\hline & HATU & & TEA & DMSO \\
\hline & HATU & & TEA & DMF/pyridine \\
\hline & Ghosez & & TEA & DCM/THF/pyridine \\
\hline & Ghosez & & TEA & DCM/THF \\
\hline & DMAP & Mukaiyama's reagent & TEA & DCM \\
\hline & DMAP & & TEA & DMA \\
\hline & DMAP & & TEA & DCM \\
\hline & DMAP & & TEA & $\mathrm{DMA} / \mathrm{CHCl}_{3}$ \\
\hline & & & TEA & DCM \\
\hline & & & TEA & DMSO \\
\hline & & & TEA & THF \\
\hline & & & TEA & $\mathrm{MeOH}$ \\
\hline & & & TEA & DMF/THF \\
\hline & & & TEA & $\mathrm{ACN} / \mathrm{H}_{2} \mathrm{O}$ \\
\hline & & & TEA & $\mathrm{MeOH} / \mathrm{THF}$ \\
\hline & & & TEA & DCM/THF \\
\hline & TBD & & & toluene \\
\hline & T3P & & & THF \\
\hline & T3P (50\% in EtOAc) & & pyridine & EtOAc \\
\hline & T3P & & pyridine & DMF \\
\hline & PYAOP & & pyridine & DMF \\
\hline & $\mathrm{POCl}_{3}$ & & & pyridine \\
\hline & $\mathrm{POCl}_{3}$ & & pyridine & DMA \\
\hline & Ghosez & & pyridine & THF \\
\hline & Ghosez & & pyridine & DCM/THF/pyridine \\
\hline & Ghosez & & pyridine & DCM/THF \\
\hline & EDC & & pyridine & DMF \\
\hline & EDC & & pyridine & DMA \\
\hline & DSC & & pyridine & ACN \\
\hline & EDC & DMAP & pyridine & DMF \\
\hline & EDC & DMAP & pyridine & DCM/DMF \\
\hline & & DMAP & pyridine & $\mathrm{DMA} / \mathrm{CHCl}_{3}$ \\
\hline & & & pyridine & DCM \\
\hline & & & & Pyridine \\
\hline & & & pyridine & DMA \\
\hline & & & pyridine & $\mathrm{DMA} / \mathrm{CHCl}_{3}$ \\
\hline & & & pyridine & THF \\
\hline${\mathrm{PS}-\mathrm{PPh}_{3}}_{3}$ & $\mathrm{CCl}_{3} \mathrm{CN}$ & & & ACN \\
\hline $\mathrm{PS}^{-\mathrm{PPh}_{3}}$ & $\mathrm{CCl}_{3} \mathrm{CN}$ & & & THF \\
\hline
\end{tabular}




\begin{tabular}{|c|c|c|c|c|}
\hline & Oxalyl chloride & & & DCM/DMF \\
\hline & & & & DMA \\
\hline & & & & DCE \\
\hline & & & & DMA/THF \\
\hline & & & & THF \\
\hline & HATU & & $\mathrm{N}$-methylmorpholine & DMF \\
\hline & DIC & Oxyma-B & Lutidine & DMF \\
\hline & HATU & & Lutidine & DMF \\
\hline & HATU & & Lutidine & DMA \\
\hline & & & KOtBu & \\
\hline & & & $\mathrm{K}_{2} \mathrm{CO}_{3}$ & \\
\hline PS-DCC & $\mathrm{HOBt}$ & & & DMA \\
\hline \multirow[t]{5}{*}{ PS-DCC } & $\mathrm{HOBt}$ & & & DMA/MeOH \\
\hline & EDC & $\mathrm{HOBt}$ & & DMA \\
\hline & HOAt & CDI & & ACN \\
\hline & Ghosez & & & DCM \\
\hline & Ghosez & & & $\mathrm{DCM} / \mathrm{THF}$ \\
\hline \multirow[t]{18}{*}{ PS-DCC } & DMAP & & & DCM \\
\hline & Ghosez & DMAP & & THF \\
\hline & EDC & DMAP & & DCM \\
\hline & EDC & DMAP & & DMF \\
\hline & EDC & DMAP & & DCE \\
\hline & EDC & DMAP & & DCM/DMF \\
\hline & & DMAP & & DMA \\
\hline & & DMAP & & THF \\
\hline & HATU & & Diisopropylamine & DMF \\
\hline & HATU & & Diisopropylamine & DMA \\
\hline & & & Diisopropylamine & dioxane \\
\hline & & & & \\
\hline & & & & \\
\hline & T3P (50\% in EtOAc) & & DIEA & DCM \\
\hline & T3P & & DIEA & DMF \\
\hline & DSC & pyridine & DIEA & ACN \\
\hline & PYAOP & & DIEA & DMF \\
\hline & PYAOP & & DIEA & DMA \\
\hline PS-TBTU & & & DIEA & DMA/DMF \\
\hline PS-PPh ${ }_{3}$ & $\mathrm{CCl}_{3} \mathrm{CN}$ & & DIEA & THF \\
\hline PS-DCC & $\mathrm{HOBt}$ & Si-Carbonate & DIEA & DMA \\
\hline PS-DCC & $\mathrm{HOBt}$ & & DIEA & ACN \\
\hline $\begin{array}{l}\text { PS-DCC } \\
\end{array}$ & HOBt & & DIEA & DMA \\
\hline PS-DCC & $\mathrm{HOBt}$ & & DIEA & DCM \\
\hline PS-DCC & $\mathrm{HOBt}$ & & DIEA & DMF \\
\hline EDC & $\mathrm{HOBt}$ & & DIEA & \\
\hline DCC & $\mathrm{HOBt}$ & & DIEA & DCM \\
\hline \multirow[t]{10}{*}{ DCC } & $\mathrm{HOBt}$ & & DIEA & DMA \\
\hline & HBTU & Si-carbonate & DIEA & DMF \\
\hline & HATU & Si-carbonate & DIEA & DMF \\
\hline & HATU & & DIEA & DCM \\
\hline & HATU & & DIEA & DMA \\
\hline & HATU & & DIEA & DMF \\
\hline & HATU & & DIEA & DCM/THF/pyridine \\
\hline & HATU & & DIEA & DMA/THF \\
\hline & & & DIEA & DMF \\
\hline & & & DIEA & DCM \\
\hline
\end{tabular}




\begin{tabular}{|l|l|l|l|l|}
\hline & & & DIEA & ACN \\
\hline & & & DIEA & dioxane \\
\hline & & & DIEA & DMA/ACN \\
\hline & & & DIEA & THF \\
\hline & & & DIEA & DCE \\
\hline & & & DIEA & DMA \\
\hline & & & DIEA & DCM/pyridine \\
\hline & & & DIEA & pyridine \\
\hline & & & DIEA & DMSO \\
\hline & TBTU & & TEA & DMA or DMF \\
\hline & TBTU & TEA & DMF \\
\hline & TBTU & Si-carbonate & DIEA & DMA \\
\hline & TBTU & Si-carbonate & DIEA & ACN \\
\hline & TBTU & & DIEA & DMA/ACN \\
\hline
\end{tabular}

Table S2. Suzuki Coupling Conditions Used in Library Synthesis

\begin{tabular}{|l|l|l|l|}
\hline Catalyst & Base & Ligand or work-up resin & Solvent(s) \\
\hline Catacxium A Pd G3 & Cs2CO3 & & toluene;water \\
\hline CX31 & K3PO4 & & THF;water \\
\hline CX31 & NaHCO3 & & THF;Water \\
\hline Fibercat & Cs2CO3 & & dioxane;water \\
\hline FibreCat 1001 & K2CO3 & Si-Carbonate & EtOH \\
\hline FibreCat 1007 & K2CO3 & Si-Carbonate & DMF \\
\hline FibreCat 1007 & K2CO3 & Si-Carbonate & EtOH \\
\hline FibreCat 1007 & K2CO3 & Si-Carbonate & EtOH;DME \\
\hline FibreCat 1032 & Cs2CO3 & & EtOH;water \\
\hline FibreCat 1032 & K2CO3 & Si-Carbonate & EtOH;water \\
\hline FibreCat 1032 & K2CO3 & & DCE;EtOH;water \\
\hline FibreCat 1032 & K2CO3 & & EtOH \\
\hline PA Ph Pd G3 & K3PO4 & & dioxane;water \\
\hline PA-Ph Pd G3 & Cs2CO3 & & dioxane;water \\
\hline PA-Ph Pd G3 & K2CO3 & & dioxane;water \\
\hline PA-Ph Pd G3 & K3PO4 & & THF;water \\
\hline Pd(Amphos)Cl2 & K2CO3 & & dioxane;water \\
\hline Pd(ddpf)Cl2 & Na2CO3 & & THF;MeOH;water \\
\hline Pd(ddpf)Cl2 & NaHCO3 & & dioxane \\
\hline Pd(DPEPhos)Cl2 & Cs2CO3 & & dioxane;water \\
\hline Pd(dppe)Cl2 & K3PO4 & & dioxane;water \\
\hline Pd(dppf)Cl2 & Cs2CO3 & & dioxane;water \\
\hline Pd(dppf)Cl2 & Cs2CO3 & EtOH \\
\hline Pd(dppf)Cl2 & Cs2CO3 & DME;water \\
\hline Pd(dppf)Cl2 & Cs2CO3 & & DMA \\
\hline Pd(dppf)Cl2 & K2CO3 & & THF \\
\hline Pd(dppf)Cl2 & K2CO3 & Toluene \\
\hline & & & \\
\hline
\end{tabular}




\begin{tabular}{|c|c|c|c|}
\hline $\mathrm{Pd}(\mathrm{dppf}) \mathrm{Cl} 2$ & $\mathrm{~K} 2 \mathrm{CO} 3$ & & dioxane;water \\
\hline $\mathrm{Pd}(\mathrm{dppf}) \mathrm{Cl} 2$ & $\mathrm{~K} 2 \mathrm{CO} 3$ & & DME;water \\
\hline $\mathrm{Pd}(\mathrm{dppf}) \mathrm{Cl} 2$ & K3P04 & & DME \\
\hline $\mathrm{Pd}(\mathrm{dppf}) \mathrm{Cl} 2$ & K3PO4 & & dioxane \\
\hline $\mathrm{Pd}(\mathrm{dppf}) \mathrm{Cl} 2$ & $\mathrm{Na} 2 \mathrm{CO} 3$ & & THF;water \\
\hline $\mathrm{Pd}(\mathrm{dppf}) \mathrm{Cl} 2$ & $\mathrm{Na} 2 \mathrm{CO} 3$ & & dioxane \\
\hline $\mathrm{Pd}(\mathrm{dppf}) \mathrm{Cl} 2$ & $\mathrm{NaHCO} 3$ & & DME;EtOH;Water \\
\hline $\mathrm{Pd}(\mathrm{dppf}) \mathrm{Cl} 2-\mathrm{CH} 2 \mathrm{Cl} 2$ & $\mathrm{Cs} 2 \mathrm{CO} 3$ & & dioxane;water \\
\hline $\mathrm{Pd}(\mathrm{dppf}) \mathrm{Cl} 2-\mathrm{CH} 2 \mathrm{Cl} 2$ & $\mathrm{Cs} 2 \mathrm{CO} 3$ & & DME \\
\hline $\mathrm{Pd}(\mathrm{dppf}) \mathrm{Cl} 2-\mathrm{CH} 2 \mathrm{Cl} 2$ & $\mathrm{~K} 2 \mathrm{CO} 3$ & & THF \\
\hline $\mathrm{Pd}(\mathrm{dppf}) \mathrm{Cl} 2-\mathrm{CH} 2 \mathrm{Cl} 2$ & $\mathrm{~K} 2 \mathrm{CO} 3$ & & dioxane \\
\hline $\mathrm{Pd}(\mathrm{dtbpf}) \mathrm{Cl} 2$ & $\mathrm{Cs} 2 \mathrm{CO} 3$ & & DMF;water \\
\hline $\mathrm{Pd}(\mathrm{dtbpf}) \mathrm{Cl} 2$ & $\mathrm{~K} 2 \mathrm{CO} 3$ & & dioxane \\
\hline $\mathrm{Pd}(\mathrm{dtbpf}) \mathrm{Cl} 2$ & K3P04 & & dioxane;water \\
\hline $\operatorname{Pd}(\mathrm{OAc}) 2$ & $\mathrm{CsF}$ & Сy-Мap & DME;MeOH \\
\hline $\mathrm{Pd}(\mathrm{OAc}) 2$ & K3P04 & SPhos & dioxane;water \\
\hline $\mathrm{Pd}(\mathrm{PPh} 3) 4$ & $\mathrm{Na} 2 \mathrm{CO} 3$ & & DME;water \\
\hline $\mathrm{Pd}(\mathrm{PPh} 3) 4$ & $\mathrm{Na} 2 \mathrm{CO} 3$ & & toluene;EtOH \\
\hline $\mathrm{Pd} 2(\mathrm{dba}) 3$ & Cs2CO3 & $\mathrm{PA}-\mathrm{Ph}$ & THF;water \\
\hline $\mathrm{Pd} 2(\mathrm{dba}) 3$ & $\mathrm{Cs} 2 \mathrm{CO} 3$ & XPhos & dioxane;water \\
\hline $\mathrm{Pd} 2(\mathrm{dba}) 3$ & K3PO4 & $\mathrm{PA}-\mathrm{Ph}$ & THF;water \\
\hline $\mathrm{Pd} 2(\mathrm{dba}) 3$ & K3PO4 & $\mathrm{PA}-\mathrm{Ph}$ & DME;water \\
\hline $\mathrm{Pd} 2(\mathrm{dba}) 3$ & K3PO4 & $\mathrm{PA}-\mathrm{Ph}$ & dioxane;water \\
\hline $\mathrm{Pd} 2(\mathrm{dba}) 3$ & K3PO4 & $\mathrm{PCy} 3$ & THF;water \\
\hline $\mathrm{Pd} 2(\mathrm{dba}) 3$ & K3P04 & PCy3 & dioxane;water \\
\hline $\mathrm{Pd} 2(\mathrm{dba}) 3$ & K3PO4 & XPhos & dioxane;water \\
\hline $\mathrm{Pd} 2(\mathrm{dba}) 3$ & $\mathrm{KF}$ & SPhos & dioxane;water \\
\hline $\mathrm{Pd} 2(\mathrm{dba}) 3$ & $\mathrm{Na} 2 \mathrm{CO} 3$ & $\mathrm{PA}-\mathrm{Ph}$ & dioxane;water \\
\hline $\mathrm{Pd} 2 \mathrm{dba} 3$ & $\mathrm{Na} 2 \mathrm{CO} 3$ & XPhos & EtOH \\
\hline $\mathrm{PdCl} 2(\mathrm{PCy} 3) 2$ & $\mathrm{Cs} 2 \mathrm{CO} 3$ & & dioxane;water \\
\hline $\mathrm{PdCl} 2(\mathrm{PCy} 3) 2$ & $\mathrm{KOtBu}$ & & dioxane \\
\hline $\mathrm{PdCl} 2(\mathrm{PPh} 3) 2$ & Cs2CO3 & Si-Carbonate & DME;water \\
\hline $\mathrm{PdCl} 2(\mathrm{PPh} 3) 2$ & Cs2CO3 & & THF;EtOH \\
\hline $\mathrm{PdCl} 2(\mathrm{PPh} 3) 2$ & $\mathrm{Cs} 2 \mathrm{CO} 3$ & & EtOH;water \\
\hline $\mathrm{PdCl} 2(\mathrm{PPh} 3) 2$ & Cs2CO3 & & dioxane \\
\hline $\mathrm{PdCl} 2(\mathrm{PPh} 3) 2$ & $\mathrm{CsF}$ & & DME;MeOH \\
\hline $\mathrm{PdCl} 2(\mathrm{PPh} 3) 2$ & $\mathrm{~K} 2 \mathrm{CO} 3$ & & THF;water \\
\hline $\mathrm{PdCl} 2(\mathrm{PPh} 3) 2$ & $\mathrm{~K} 2 \mathrm{CO} 3$ & & DME;water \\
\hline $\mathrm{PdCl} 2(\mathrm{PPh} 3) 2$ & $\mathrm{~K} 2 \mathrm{CO} 3$ & & EtOH;water \\
\hline $\mathrm{PdCl} 2(\mathrm{PPh} 3) 2$ & $\mathrm{~K} 2 \mathrm{CO} 3$ & & dioxane;water \\
\hline $\mathrm{PdCl} 2(\mathrm{PPh} 3) 2$ & $\mathrm{~K} 2 \mathrm{CO} 3$ & & DME;EtOH;water \\
\hline
\end{tabular}




\begin{tabular}{|c|c|c|c|}
\hline PdCl2(PPh3)2 & $\mathrm{K} 2 \mathrm{CO} 3$ & & MeOH;THF;water \\
\hline $\mathrm{PdCl} 2(\mathrm{PPh} 3) 2$ & $\mathrm{~K} 2 \mathrm{CO} 3$ & & dioxane;EtOH;water \\
\hline $\mathrm{PdCl} 2(\mathrm{PPh} 3) 2$ & $\mathrm{Na} 2 \mathrm{CO} 3$ & & DME;water \\
\hline PdEnCat & $\mathrm{K} 2 \mathrm{CO} 3$ & & ACN; water \\
\hline PEPPSI & $\mathrm{Cs} 2 \mathrm{CO} 3$ & & DME;H2O \\
\hline PEPPSI-iPr & $\mathrm{tBuOK}$ & & IPA \\
\hline PS-P(Ph)3-Pd & $\mathrm{K} 2 \mathrm{CO} 3$ & Si-Carbonate & EtOH/DME \\
\hline PS-P(Ph)3-Pd & $\mathrm{K} 2 \mathrm{CO} 3$ & & dioxane \\
\hline PS-P(Ph)3-Pd & $\mathrm{Na} 2 \mathrm{CO} 3$ & & DME \\
\hline RuPhos Pd G3 & $\mathrm{Cs} 2 \mathrm{CO} 3$ & & Dioxane \\
\hline SiliaCat & $\mathrm{Cs} 2 \mathrm{CO} 3$ & & Dioxane \\
\hline SiliaCat & $\mathrm{Cs} 2 \mathrm{CO} 3$ & & EtOH;water \\
\hline SiliaCat & $\mathrm{Cs} 2 \mathrm{CO} 3$ & & THF;EtOH;water \\
\hline Siliacat Pd-DPP & $\mathrm{Cs} 2 \mathrm{CO} 3$ & & EtOH;water \\
\hline SiliaCat Pd-DPP & $\mathrm{Cs} 2 \mathrm{CO} 3$ & & dioxane;water \\
\hline Siliacat Pd-DPP & $\mathrm{K} 2 \mathrm{CO} 3$ & & DME;H2O \\
\hline SiliaCat Pd-DPP & $\mathrm{NaHCO} 3$ & & iPrOH;water \\
\hline Silicat Pd-dppf & Cs2CO3 & & EtOH;water \\
\hline SPhos Pd G3 & $\mathrm{Cs} 2 \mathrm{CO} 3$ & & DMF;dioxane;water \\
\hline SPhos Pd G3 & K3PO4 & & dioxane;water \\
\hline Xantphos Pd G3 & Cs2CO3 & & \\
\hline XPhos Pd G2 & $\mathrm{K} 2 \mathrm{CO} 3$ & XPhos & EtOH;water \\
\hline XPhos Pd G3 & $\mathrm{Cs} 2 \mathrm{CO} 3$ & & dioxane;water \\
\hline XPhos Pd G3 & K3PO4 & $\mathrm{Cu}(\mathrm{OAc})_{2}$ & DMA \\
\hline XPhos Pd G3 & K3P04 & & dioxane; water \\
\hline
\end{tabular}


Table S3. Common C-N Coupling Library Reaction Conditions Used with Different Substrates

\begin{tabular}{|c|c|c|c|c|c|}
\hline Substrate 1 & Substrate 2 & Catalyst & Ligand & Base & Solvent \\
\hline \multirow{6}{*}{ (hetero)aryl-X } & \multirow{6}{*}{$\begin{array}{l}\text { aniline } \\
\text { (1.5 equiv) }\end{array}$} & \multirow{4}{*}{$\mathrm{Pd}_{2}(\mathrm{dba})_{3}$} & \multirow{4}{*}{ XPhos } & $\mathrm{K}_{3} \mathrm{PO}_{4}$ (3 equiv) & 1,4 dioxane \\
\hline & & & & $\mathrm{Cs}_{2} \mathrm{CO}_{3}$ (3 equiv) & 1,4 dioxane \\
\hline & & & & $\mathrm{K}_{3} \mathrm{PO}_{4}$ (3 equiv) & t-amylOH \\
\hline & & & & $\mathrm{Cs}_{2} \mathrm{CO}_{3}$ (3 equiv) & t-amylOH \\
\hline & & BrettPhos Pd G3 & BrettPhos & $\mathrm{NaOtBu}$ (2 equiv) & DME \\
\hline & & BrettPhos Pd G3 & BrettPhos & LiHMDS ( 2 equiv) & DME \\
\hline (hetero)aryl-X & $\begin{array}{l}\text { heteroaryl } \\
\text { aniline } \\
\text { (1.5 equiv) }\end{array}$ & $\mathrm{Pd}_{2}(\mathrm{dba})_{3}$ & XPhos & $\mathrm{Cs}_{2} \mathrm{CO}_{3}$ (3 equiv) & 1,4 dioxane \\
\hline (hetero)aryl-X & $\begin{array}{l}\text { primary amine } \\
\text { (1.5 equiv) }\end{array}$ & RuPhos Pd G1 & RuPhos & LiHMDS (2 equiv) & 1,4 dioxane \\
\hline \multirow{2}{*}{$\begin{array}{l}\text { heteroaryl } \\
\text { (Br/Cl), pyridines } \\
\text { (Cl/Br) and } \\
\text { quinolines }\end{array}$} & \multirow{2}{*}{$\begin{array}{l}\text { primary amine } \\
\text { (1.5 equiv) }\end{array}$} & \multirow[b]{2}{*}{ BrettPhos Pd G3 } & - & $\mathrm{NaOtBu}$ (1.5 equiv) & THF \\
\hline & & & BrettPhos & $\mathrm{NaOtBu}$ (2 equiv) & DME \\
\hline $\begin{array}{l}\text { heteroaryl } \\
\text { (Br/Cl), pyridines } \\
\text { (Cl/Br) and } \\
\text { quinolines }\end{array}$ & $\begin{array}{l}\text { substituted } \\
\text { primary amine } \\
\text { ( } 2 \text { equiv) }\end{array}$ & tBuXPhos Pd G3 & - & $\mathrm{NaOtBu}$ (2.5 equiv) & $\mathrm{tBuOH}$ \\
\hline (hetero)aryl-X & $\begin{array}{l}\text { secondary } \\
\text { amine } \\
\text { (1.5 equiv) }\end{array}$ & $\mathrm{Pd}_{2}(\mathrm{dba})_{3}$ & RuPhos & $\begin{array}{l}\text { NaOtBu (2 equiv) or } \\
\text { LiHMDS ( } 1.5 \text { equiv) }\end{array}$ & THF \\
\hline (hetero)aryl-X & $\begin{array}{l}\text { secondary } \\
\text { amine } \\
\text { (1.5 equiv) }\end{array}$ & tBuXPhos Pd G3 & - & $\mathrm{NaOtBu}$ (2 equiv) & $t \mathrm{BuOH}$ \\
\hline $\begin{array}{l}\text { aryl, quinoline, } \\
\text { and hindered } \mathrm{Br}\end{array}$ & $\begin{array}{l}\text { protected } \\
\text { piperazine }\end{array}$ & $\mathrm{Pd}_{2}(\mathrm{dba})_{3}$ & XPhos & $\mathrm{NaOtBu}$ (2 equiv) & DME \\
\hline aryl-I & $\begin{array}{l}\text { protected } \\
\text { piperazine }\end{array}$ & RuPhos Pd G1 & - & $\mathrm{NaOtBu}$ (3 equiv) & THF \\
\hline $\begin{array}{l}\text { heteroaryl-X (2,6- } \\
\text { dichloro pyridine) }\end{array}$ & $\begin{array}{l}\text { sterically } \\
\text { hindered } \\
\text { primary amine }\end{array}$ & $\mathrm{Pd}_{2}(\mathrm{dba})_{3}$ & APhos & $\mathrm{Cs}_{2} \mathrm{CO}_{3}(3$ equiv $)$ & 1,4 dioxane \\
\hline hindered aryl-X & aniline & tBuXPhos Pd G3 & - & $\mathrm{NaOtBu}$ (2 equiv) & $t \mathrm{BuOH}$ \\
\hline $\begin{array}{l}\text { hindered } \\
\text { heteroaryl-Cl }\end{array}$ & $\begin{array}{l}\text { aniline, } \\
\text { heteroaryl- } \\
\text { benzylamine }\end{array}$ & tBuXPhos Pd G3 & - & $\mathrm{NaOtBu}$ (2.3 equiv) & $t \mathrm{BuOH}$ \\
\hline hindered aryl-Br & $\begin{array}{l}\text { secondary } \\
\text { amine }\end{array}$ & $\begin{array}{l}\text { dichloro[1,3-bis(2,6-di-3-pentylp } \\
\text { henyl)imidazol-2-ylidene](o-picol } \\
\text { ine)palladium(II) (PEPPSI- } \\
\text { IPent }^{\mathrm{Cl}} \text {-o-picoline) }\end{array}$ & - & $\mathrm{KOtBu}$ & DME \\
\hline heteroaryl-Br/Cl & $\begin{array}{l}\text { secondary } \\
\text { amine }\end{array}$ & PEPPSI-IPent & - & $\mathrm{KOtBu}$ & DME \\
\hline $\begin{array}{l}\text { electron rich } \\
\text { aryl-Br }\end{array}$ & primary amine & $\begin{array}{l}\text { dichloro[1,3-bis(2,6-di-3- } \\
\text { pentylphenyl)imidazol-2- } \\
\text { ylidene](3- } \\
\text { chloropyridyl)palladium(II) } \\
\text { (PEPPSI-IPent }{ }^{\mathrm{Cl}} \text { ) }\end{array}$ & - & $\mathrm{KOtBu}$ & DME \\
\hline
\end{tabular}


